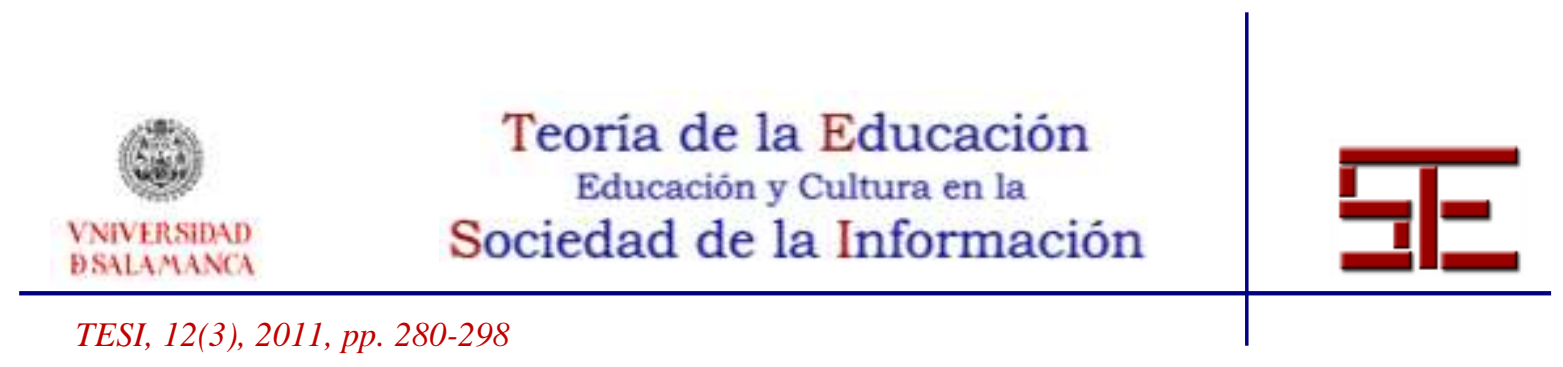

\title{
LA GENERACIÓN NET SE TAMBALEA: PERCEPCIÓN DEL DOMINIO DE LAS TIC DE ESTUDIANTES DE MAGISTERIO
}

Resumen: Distintos autores aseguran que los alumnos universitarios nacidos después de 1982 muestran unas características diferentes a las generaciones anteriores. Se suele suponer que dichos alumnos, denominados la "Generación Net", se muestran más predispuestos a dominar las tecnologías y las integran en todos los aspectos de sus vidas, pero ¿Podemos observar dicha predisposición en nuestros alumnos?

El objetivo de este artículo consiste en descubrir si existe alguna diferencia significativa en la percepción de las competencias relacionadas al dominio de las Tecnologías de la Información y la Comunicación (TIC) entre alumnos de magisterio nacidos antes de los años 80 y aquellos alumnos que nacieron después de esta fecha. Para llevar a cabo dicho estudio se empleó un estudio de correlación entre la edad de un grupo de alumnos de magisterio y su percepción acerca del dominio de diferentes herramientas TIC. Los datos del estudio se recogieron a través de cuestionarios validados por expertos.

El análisis de los datos no muestra una relación significativa entre la mayoría de variables analizadas. No obstante, indica una percepción del uso hábil de las herramientas de edición de vídeo y redes sociales por parte de los participantes más jóvenes. Se argumenta que la educación contemporánea, con demasiada frecuencia basada en las formas de aprendizaje verbales por escrito, necesita reorientar su enfoque con el fin de motivar a las generaciones más jóvenes.

Palabras clave:Educación superior; TIC; alumnado; formación de maestros; análisis cuantitativo; alfabetización digital; competencias TIC; Web 2.

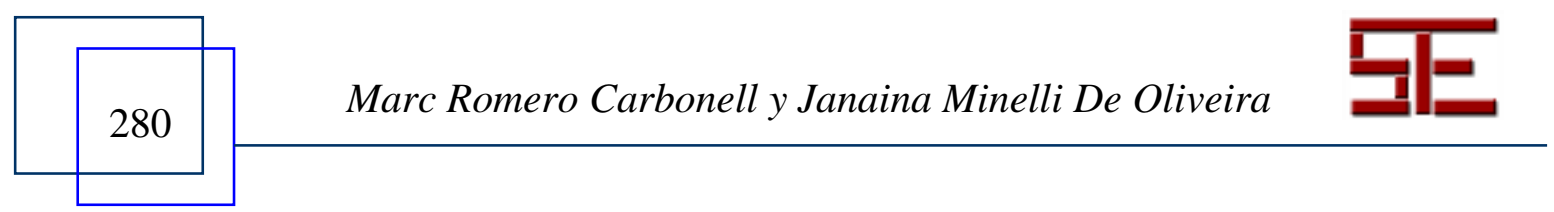




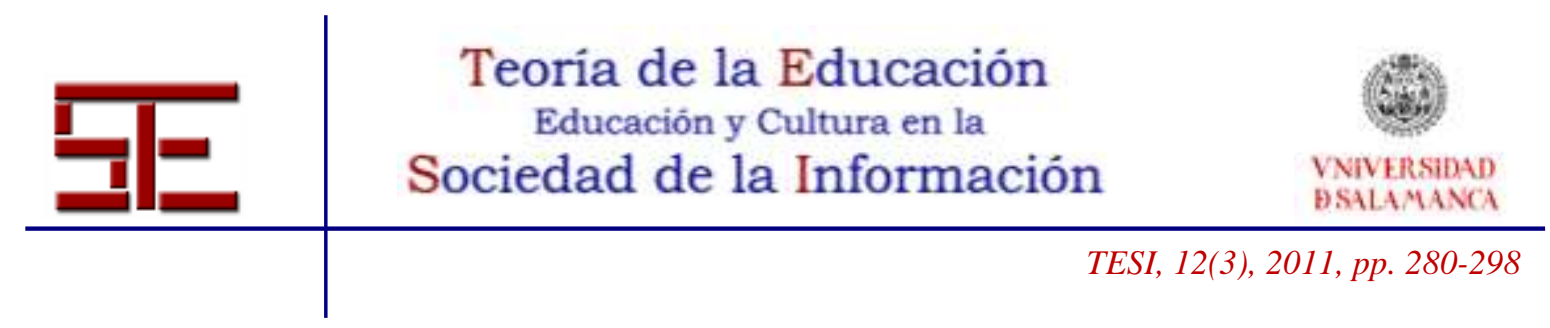

\section{THE WOBBLY NET GENERATION: STUDENT TEACHERS' PERCEPTION OF THEIR ICT SKILLS}

Abstract: Many authors have stated that undergraduate students born after 1982 show different characteristics when compared to previous generations. It is often assumed that these students, called the "Net Generation", are more predisposed to master the technologies and integrate them into all aspects of their lives. However, it is relevant to ask if that is an actual observable phenomenon. Are students born after the 80s really more kin of ICT tools than those born in previous generations?

The objective of this present research is to investigate the existence of significant differences in the perceptions of Information and Communication Technology (ICT) related skills of student teachers born before and after the 80s. In order to achieve such an objective, we performed a correlational study, holding the participants' age and level of perceived control of different ICT tools as variables. The research data was collected through expert validated questionnaires.

Data analysis shows no significant relationship between most variables analyzed. Nevertheless, it indicates perception of skillful use of video editing tools and social networks by younger participants. It is argued that the contemporary education, too often mostly based in verbal written forms of learning and teaching, needs reorientation in focus so as to motivate the younger generation.

Keywords: Higher Education; ICT; students; teacher training; quantitative analysis; digital literacy; ICT competences; Web 2.

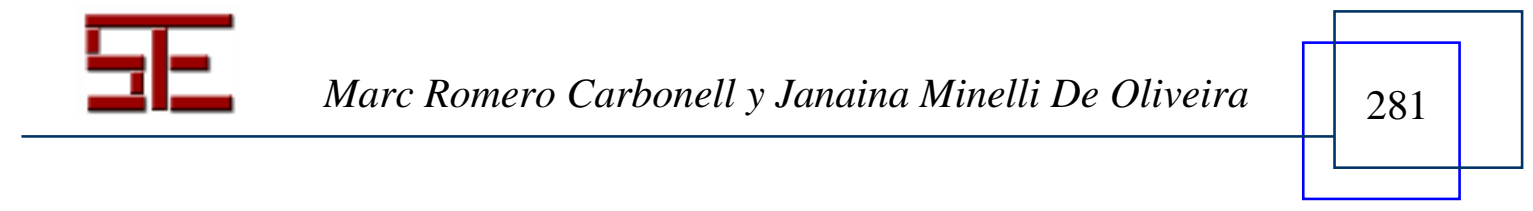




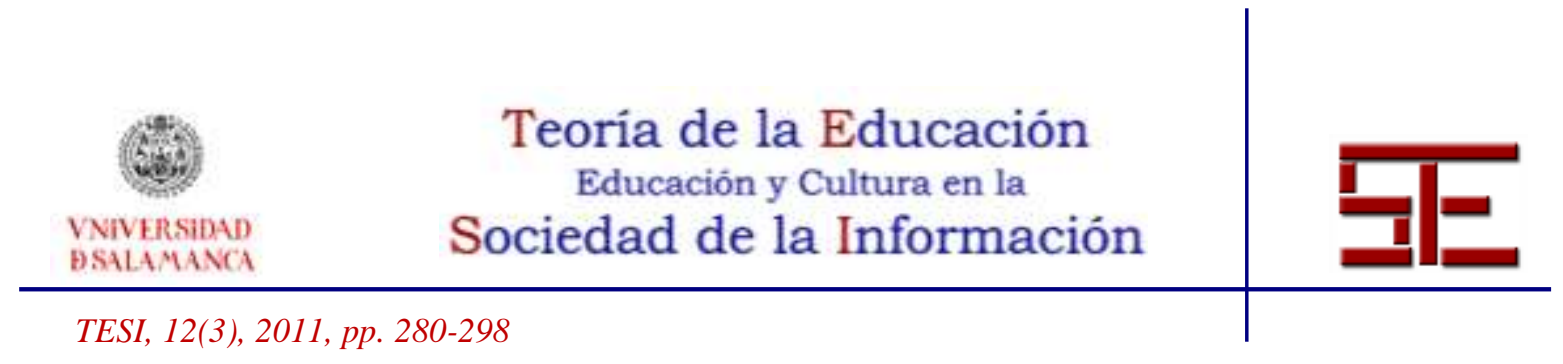

\section{LA GENERACIÓN NET SE TAMBALEA. PERCEPCIÓN DEL DOMINIO DE LAS TIC DE ESTUDIANTES DE MAGISTERIO}

Fecha de recepción: 29/07/2011; fecha de aceptación: 13/09/2011; fecha de publicación: 30/11/2011

Marc Romero Carbonell

mromerocar@uoc.edu

Universitat Oberta de Catalunya

Janaina Minelli De Oliveira

janaina.oliveira@urv.cat

Universitat Rovira i Virgili

\section{1.- INTRODUCCIÓN}

En la actualidad, existe una corriente (Oblinger y Oblinger, 2005; Palfrey y Gasser, 2008; Prensky, 2005; Tapscott, 2009), sobre todo en Estados Unidos, que asegura la existencia de una nueva generación que ha vivido su infancia rodeada de los medios de comunicación y la informática, por lo que poseen un conocimiento y dominio de las TIC más intuitivo y profundo que las anteriores generaciones. Este hecho se supone que también afecta a su aprendizaje, dado que se les atribuye un esquema de pensamiento distinto (Tapscott, 1998). Desde esta perspectiva, la gran mayoría de los alumnos universitarios, con edades que varían entre los 18 y 30 años, podrían ser considerados dentro de lo que se llama Generación Net, ya que "se trata de aquellos que nacieron a partir del año 1980" (Oblinger y Oblinger, 2005, 12).

Según la literatura analizada, la Generación Net es aquella que ha estado desde su primera infancia en contacto con las TIC, por lo que su bagaje en el uso y dominio de éstas les permite adaptarse de forma rápida y eficiente a los cambios que implican a varios niveles (Social, económico, académico...) las revoluciones tecnológicas.

Aparte de la Generación Net, existen varias denominaciones que intentan delimitar tanto su contexto cronológico como características, planteando el hecho de que ha tenido las TIC a su alcance desde edades muy tempranas. Una de las más conocidas es la de Nativos Digitales, definidos como aquellos que "son habladores nativos del lenguaje digital de los ordenadores, los videojuegos e Internet" (Prensky, 2001, 1); denominando "Inmigrantes Digitales" a aquellos nacidos antes de los años 80.

Algunos de los autores mencionados les atribuyen una serie de características (Oblinger y Oblinger, 2005; Dede, 2005) como el hecho de estar digitalmente alfabetizados, continuamente conectados, mostrando una necesidad de inmediatez en la recepción de

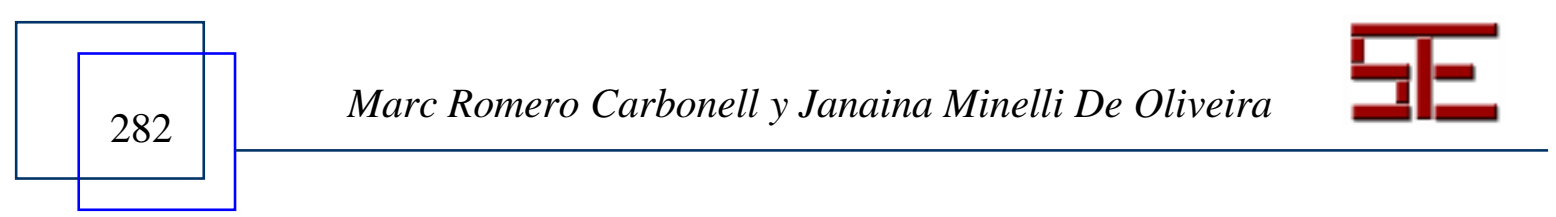




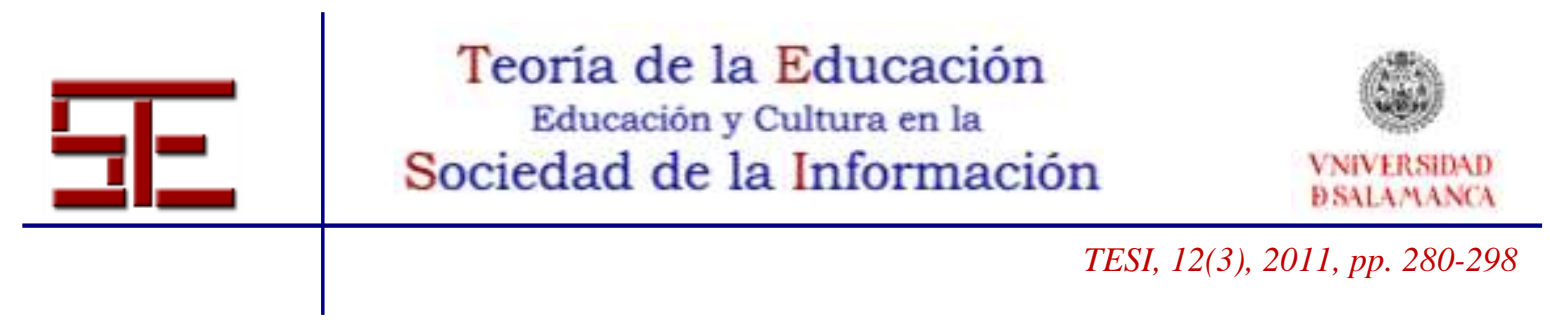

información, preferencia por las actividades sociales, una preferencia por las tareas de carácter experimental, una capacidad de llevar a cabo diversas tareas de forma simultánea y comprometidos con la comunidad. Pero, ¿hasta qué medida esta generación muestra las características que la literatura actual les atribuye?

Diversos estudios (Kennedy y otros, 2008; Bennet, Maton y Kervin, 2008; Xiaoqing y otros, 2008; Selwyn, 2009; Romero y otros, 2010; Bullen, Morgan y Qayyum, 2011) ponen de manifiesto que existe una importante laguna científica a la hora de demostrar las afirmaciones más importantes de esta generación. De hecho, algunos de los autores citados refutan sus características más conocidas argumentando que también pueden mostrarse en otras generaciones: "No existen evidencias de que la capacidad de multitarea sea exclusiva de la Generación Net, de hecho, es conocida la costumbre de otras generaciones de hacer los deberes delante del televisor" (Bennet, Maton y Kervin, 2008, 779) o incluso su validez: "Diferentes estudios fallan a la hora de encontrar evidencias que den veracidad a las afirmaciones sobre el uso radicalmente distinto de las tecnologías por parte de los estudiantes más jóvenes o que manifiestan una serie de características que los diferencian de otras generaciones" (Margaryan y Littlejohn, 2011).

Por lo tanto, los jóvenes que formaron parte de los estudios mencionados están lejos de ser altamente capacitados en el uso de tecnología, conectados, socialmente ligados y con impaciencia a la hora de llevar a cabo actividades de aprendizaje pasivas.

Además, dicha sobreexposición a los medios tecnológicos no siempre puede ser considerado desde un punto de vista positivo: "El hecho de tener tanto acceso al ciberespacio puede establecer desequilibrio en el proceso cerebral, generando una sobrecarga que puede reducir la capacidad de los jóvenes de dar sentido a aquello que se les presenta o de responder de forma juiciosa" (Berson, 2003, 3).

Cada vez más parece demostrarse que afirmaciones como las de Tapscott se basan en la edad como único elemento diferenciador, considerando a los jóvenes como un cuerpo homogéneo de forma arbitraria (Lee, 2005; Hargittai, 2010). Este hecho es discutido por la aparición de estudios que afirman que existe una gran variación en el dominio y uso de la tecnología en un mismo rango de edad en las muestras elegidas (Kennedy y otros, 2008). Por lo tanto, debería ser un aspecto generacional en la que la edad es un elemento más (Oblinger y Oblinger, 2005).

Existen algunos estudios (Conole y otros, 2006) que dan apoyo a lo que la literatura dice acerca de esta generación en el ámbito educativo. Aunque en el caso de la educación superior, las críticas señaladas se evidencian de forma más palpable dado que, en primer lugar, el hecho de usar la tecnología en el ámbito educativo no implica un mayor dominio de ésta: "La exposición a los sistemas informáticos en la educación

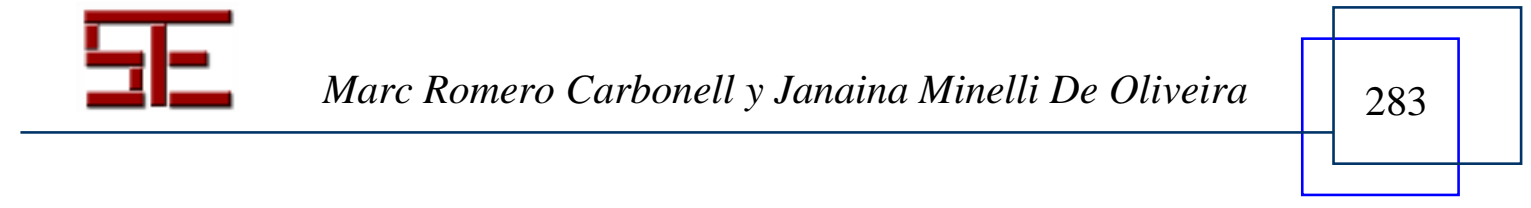




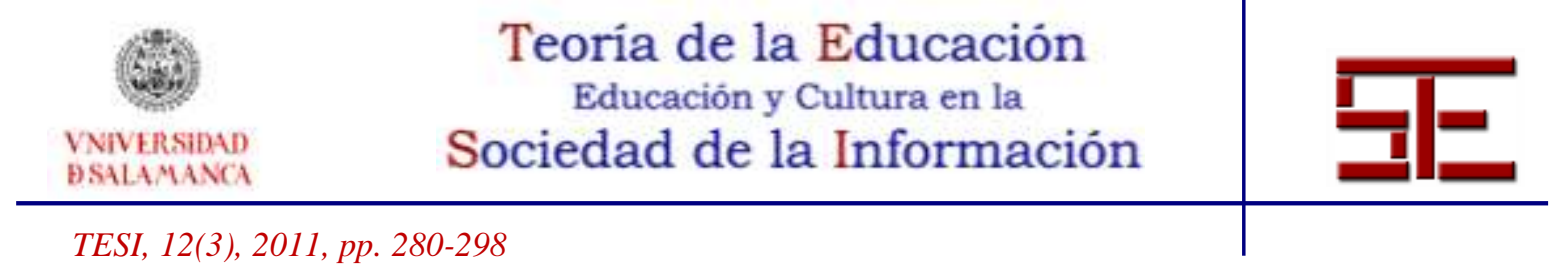

secundaria o el nivel universitario tiene muy poco nivel de impacto sobre la alfabetización digital de los alumnos" (Karsten y Roth, 1998, 15). Y, en segundo lugar, el hecho de poseer amplias habilidades en el uso de las TIC no implica su transferencia en el ámbito académico: "El uso de la tecnología para la realización de trabajos en la universidad no va más allá de su uso para un fin concreto, el hecho de ser e-competentes no significa que dicha competencia se transfiera en e-métodos para el aprendizaje" (Schulmeister, 2009).

La mayoría de estudios acerca de las características educativas de la Generación Net ponen de manifiesto que los estudiantes consideran que el uso de la tecnología en las universidades no es algo imprescindible (Bennet y otros, 2008), de hecho, los estudiantes participantes en algunos de los estudios citados, lejos de pedir a los profesores que cambien sus prácticas, parecen conformarse con las pedagogías tradicionales, aunque con menores usos de herramientas tecnológicas que muestren contenidos (Margaryan y Littlejohn, 2011).

Ante la publicidad creciente sobre las características de la Generación Net y el debate generado acerca de diferencias de esta generación en comparación con las anteriores, en este artículo se intenta descubrir si existe alguna diferencia significativa en las competencias TIC de aquellos alumnos nacidos antes de los años 80 y aquellos que nacieron después, en el marco de una investigación centrada en el estudio de las Competencias TIC de los estudiantes de la asignatura "Nuevas Tecnologías Aplicadas a la Educación" en magisterio de la Universidad Rovira i Virgili de Tarragona.

El objetivo principal de nuestra investigación es el de verificar si existe una relación significativa entre la edad de los alumnos y su percepción acerca del dominio de las TIC, concretándose en las siguientes preguntas de investigación:

¿Existe alguna diferencia en la percepción de los estudiantes de magisterio nacidos antes y después de 1982 respecto a su grado de dominio de las TIC o su forma de utilizarlas?

¿Qué impacto tiene la asignatura Nuevas Tecnologías Aplicadas a la educación en la percepción de su dominio de las TIC?

¿Es posible apreciar diferencias significativas de dicho impacto entre las dos muestras observadas?.

\section{2.- MATERIALES Y MÉTODOS}

La presente investigación empleó una metodología cuantitativa, por lo que se sitúa en el paradigma positivista (racionalista, cuantitativo) cuya principal finalidad es la de "explicar y verificar determinadas teorías o leyes para regular un fenómeno" (Arnal, Del Rincón y Latorre $(1992,43)$; dado que lo que se pretende en dicho estudio es comprobar

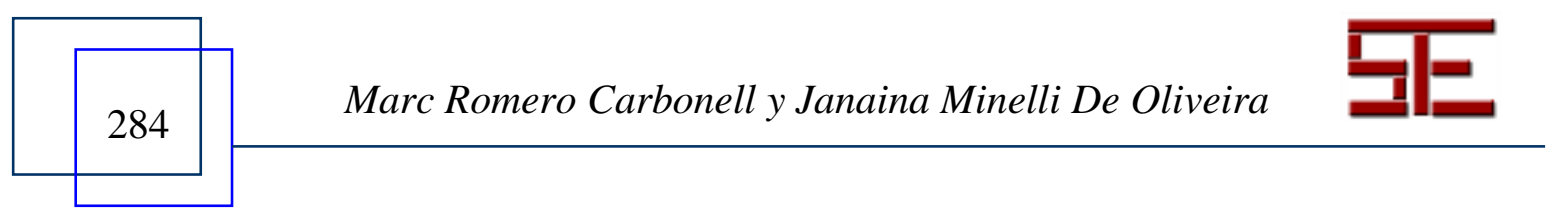




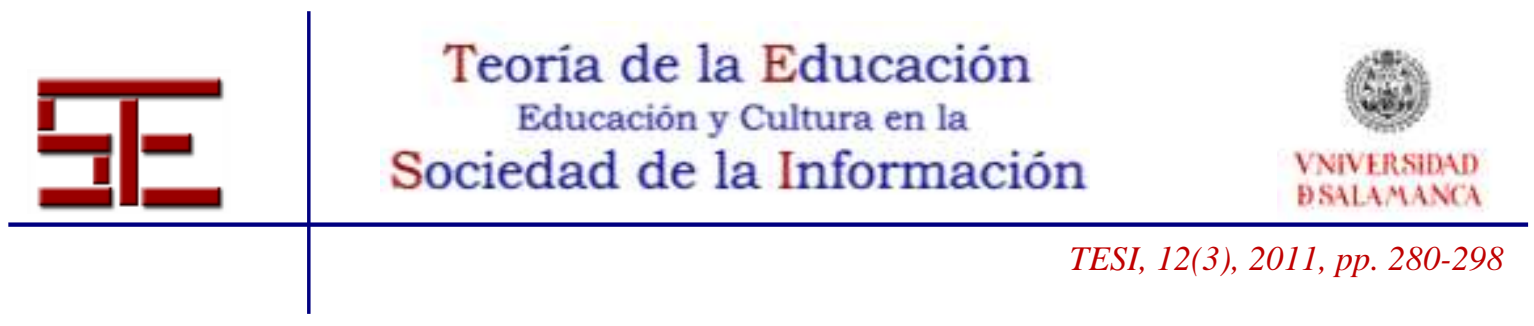

el grado de veracidad de la teorías sobre la Generación Net en una muestra de estudio determinada.

El estudio expuesto en este artículo consiste en una investigación descriptiva, en el sentido de que "el investigador describe qué ha sucedido durante el proceso de investigación" (Cohen y Manion, 1990, 101).

Los estudios descriptivos "implican la descripción detallada de las características de un fenómeno educativo" (Gall y otros, 1996, 175), dado que esta investigación se basa en la descripción de las características y percepciones de un grupo de personas con relación a un determinado fenómeno.

Siguiendo a Selltiz (1980), Deobold y otros (1981), McMillan (2004, 2006) y Lodico y otros (2006), se trata, concretamente, de un estudio de correlación, en el sentido que trata de identificar las relaciones que existen entre los hechos para lograr una verdadera comprensión del fenómeno a estudiar. De hecho, la intención es la de "demostrar si dos o más variables tienen relación mediante el cálculo de uno o más coeficientes de correlación" (McMillan, 2004, 183).

El análisis de los datos extraídos ha contemplado "la relación estadística entre una o más variables independientes con diversas variables dependientes" (McMillan, 2006, 13), que, en el caso del dicha investigación, son el año de nacimiento de los sujetos de estudio y su percepción acerca de su dominio de las TIC.

Cabe señalar que, con la finalidad de enriquecer los resultados y posteriores conclusiones del estudio, el análisis de datos también contempla otras medidas basadas también en la estadística descriptiva.

\section{1.- Cuestionario}

El cuestionario fue el instrumento de recogida de datos elegido. Dicho cuestionario fue distribuido online al inicio y al final de la acción formativa con el objetivo de conocer la percepción de los estudiantes acerca de su grado de capacitación digital y de su uso de las TIC en actividades académicas y sociales. Se estructura en información demográfica (edad y sexo), disponibilidad de recursos TIC en el lugar de estudio y en 45 ítems valorados en una escala de Likert del 1 al 6 acerca de su capacitación digital en diversos ámbitos (dominio del ordenador, búsqueda de información, uso de herramientas ofimáticas y herramientas Web 2.0, etc.).

El cuestionario se basó en la adaptación del instrumento utilizado por Romero (2008) que fue validado a partir de una prueba piloto llevada a cabo a partir de las siguientes fases:

- Se analizó el cuestionario utilizado en el estudio CREDEFIS (Centro de Recursos Virtual de Educación Física) (Gisbert, Lutfi y Marqués, 2004) que había sido

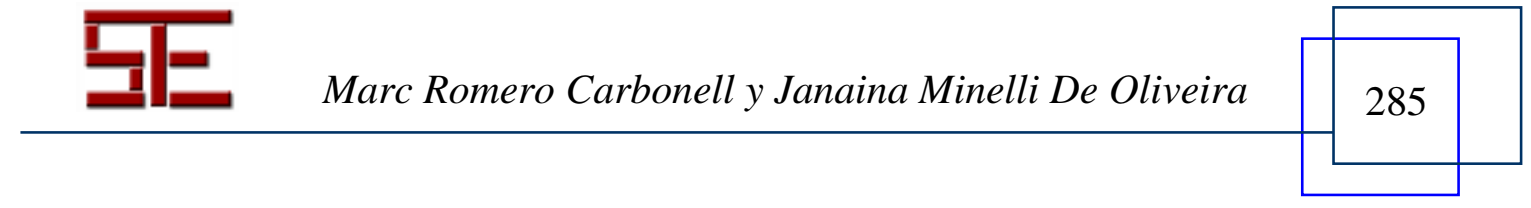




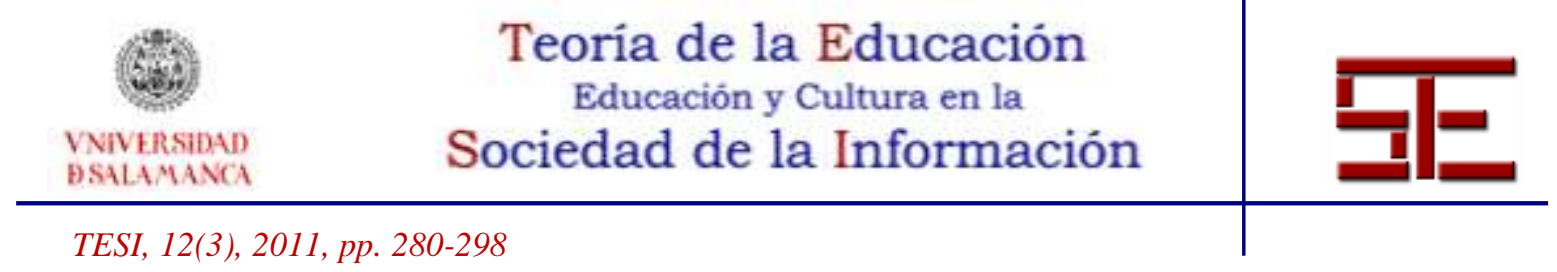

validado mediante un criterio de jueces. En dicho cuestionario se analizaban los conocimientos en TIC de los estudiantes de magisterio de la URV.

- $\quad$ Se procedió a la adaptación para dicho estudio: básicamente fueron cambiadas las preguntas basadas en una escala de valoración tipo Likert (añadiendo ítems para adaptarlo a las TIC actuales).

- $\quad$ El instrumento adaptado fue pasado a un grupo de alumnos y, a partir de los datos obtenidos, se llevó a cabo el test estadístico de consistencia interna Alpha de Cronbach en aquellos ítems valorados mediante una escala de Likert con un índice de fiabilidad del 0,89.

Con la finalidad de asegurar la fiabilidad en la actualización del cuestionario para nuestro estudio, se repitió la validación estadística del instrumento: una vez pasado el cuestionario inicial, se validaron sus preguntas a partir del coeficiente alfa de Cronbach (Cronbach, 1951) siguiendo el procedimiento descrito por Marcelo (1996) y Cabero (1998). A partir de la realización de dicha prueba, se consiguió un nivel de consistencia interna aceptable $(0,7)$ mediante SPSS 15 para Windows, por lo que no se realizaron cambios significativos en el cuestionario final.

\section{2.- Población}

Nuestro cuestionario fue distribuido entre los estudiantes de la asignatura "Nuevas Tecnologías Aplicadas a la Educación" del curso 2008/09 y podía ser cumplimentado de forma voluntaria. Se les informó de que su participación no influiría en sus calificaciones de la asignatura y se aseguró la confidencialidad de los datos recogidos.

\section{3.- Muestreo}

De los 164 estudiantes que fueron invitados a responder, se recogieron finalmente 65 cuestionarios cumplimentados por completo $\mathrm{y}$, con la finalidad de comprobar la existencia de diferencias significativas entre los estudiantes más jóvenes y los nacidos anteriormente, se seleccionaron los casos extremos, constituyendo un total de 54 individuos. Para llevar a cabo la criba según la edad, nos basamos en la perspectiva de Howe y Strauss (2000) y Schulmeister (2009), al identificar la nueva generación con aquellos que nacieron en 1982 y que se graduaron en el instituto al entrar en el nuevo milenio.

Dicha muestra supone un 32,9\% de los estudiantes del último curso de magisterio de la universidad, adquiriendo un grado de significación estadística aceptable. Todos ellos tienen unas edades comprendidas de 21 a 43 años, por lo que se han clasificado en

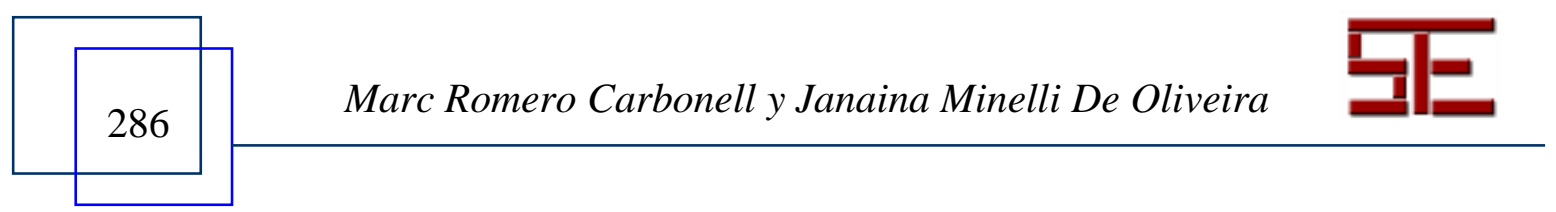




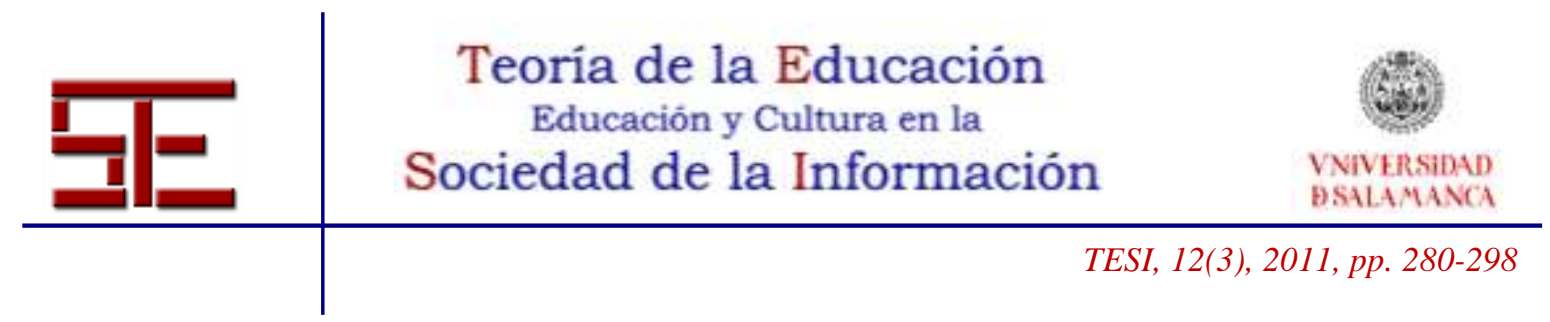

alumnos nacidos a partir de 1982 (34 individuos, un 62\% de la muestra seleccionada) y alumnos nacidos antes de 1982 (20 individuos, un 48\% de la muestra seleccionada).

En los dos grupos divididos el número de mujeres supera al de los hombres (con una ratio $90,10 \%$ )

Para llevar a cabo esta investigación, se analizaron los datos recogidos mediante pruebas estadísticas de tendencia central y el análisis de varianza multivariante (MANOVA). Para determinar la relación de la edad de la muestra (variable independiente) con los distintos ítems del cuestionario que identifican la percepción de los alumnos acerca de su grado de capacitación digital (variables dependientes). Para realizar dicho análisis se ha utilizado el paquete de análisis estadístico SPSS para Windows en su versión 15.

\section{3.- RESULTADOS}

A partir de los cuestionarios implementados al inicio y al final de la acción formativa, se extrajeron los datos referentes a la percepción de los estudiantes de su grado de dominio de las TIC divididos en seis áreas y valorados mediante una escala de Likert del 1 al 6. Se llevó a cabo el análisis de las diferencias entre los estudiantes nacidos antes y después de 1982.

\section{1.- Disponibilidad TIC}

En cuanto a la disponibilidad de TIC en el lugar de estudio, cabe señalar que en los dos casos muestran una frecuencia del 100\% tanto de ordenador como de Internet.

Los dos grupos muestran, al inicio del curso, una media de horas de uso del ordenador e Internet similares: los estudiantes nacidos después de 1982 dedican una media de 3,12 horas a la semana y los nacidos antes una media de 3,14 horas por semana. Lo mismo pasa en el final del curso, mostrando un ligero aumento en la media de horas, probablemente a causa de la realización de las actividades de la asignatura (3,65 en el caso de los alumnos nacidos después de 1982 y 3,54 en el caso de los nacidos antes).

\section{2.- Percepción del dominio general de las TIC y sus competencias digitales}

En el caso de aquellos ítems en los que los alumnos valoraban su dominio de las TIC y sus competencias digitales, se pueden observar los siguientes resultados:

El grado de valoración de su dominio del ordenador muestra, en el cuestionario inicial, un resultado $\mathrm{F}(1,53)=1,566, \mathrm{p}>.05$ por lo que no se puede afirmar que su relación con la edad sea estadísticamente significativa.

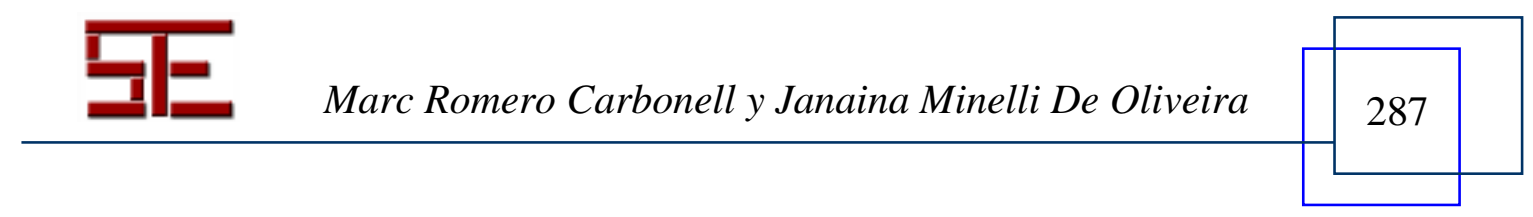




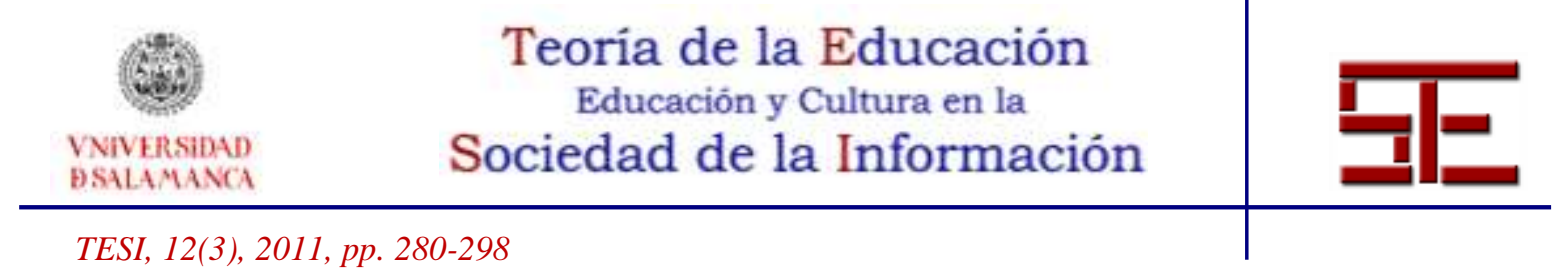

Su interés por actualizar los conocimientos sobre las TIC manifiesta un MANOVA F $(1,53)=0,457, \mathrm{p}>$. 05 por lo que su relación con la edad no es estadísticamente significativa.

Por lo tanto, no existe relación directa entre la edad de los sujetos y su percepción de su dominio e interés de actualización de sus conocimientos sobre las TIC, hecho que tampoco cambia en el cuestionario final.

\section{3.- Percepción del dominio de las herramientas ofimáticas}

El uso de herramientas ofimáticas muestra los siguientes resultados

\begin{tabular}{|l|l|l|} 
& $\mathrm{F}(1,53)$ & $\mathrm{P}$ \\
\hline Dominio Procesador de textos & 0,001 & $\mathrm{p}>.05$ \\
Dominio Generador de presentaciones & 0,886 & $\mathrm{p}>.05$ \\
Dominio de Bases de Datos & 1,619 & $\mathrm{p}>.05$ \\
Dominio de Hoja de Cálculo & 4,277 & $\mathrm{p}<.05$
\end{tabular}

Tabla 1: Resultados del análisis MANOVA entre los ítems referentes a la percepción del dominio las herramientas ofimáticas y la edad de los sujetos.

Tal y como muestra la Tabla 1, no existe una relación significativa entre la edad y su percepción acerca del dominio de la mayoría de las herramientas ofimáticas, hecho que demuestran sus frecuencias dado que el mayor número de individuos (alrededor del $56 \%$ ) puntúan la mayoría de ítems con un 1, a excepción del procesador de textos, en el que ocurre todo lo contrario ( un $61 \%$ de estudiantes nacidos antes de 1982 puntúa su dominio con un 6, mientras que un $64 \%$ lo hace en el caso de los más jóvenes). En el caso de la hoja de cálculo, puede comprobarse cierto grado de significación en su relación con la edad de los alumnos; aun así, las frecuencias indican lo contrario, dado que la mayoría de alumnos de los dos grupos puntúan su dominio con un 1.

La correspondencia entre la percepción sobre el dominio de herramientas ofimáticas y la edad de los alumnos se mantiene al final del curso, dado que no se observan cambios significativos en dicha área. Además, la ligera significación de la relación entre la edad y la percepción sobre el dominio de las hojas de cálculo desaparece $(\mathrm{F}(1,53)=1,680$ $\mathrm{p}>.05)$.

\section{4.- Percepción del dominio de herramientas de edición multimedia}

Los encuestados mostraron ciertas diferencias en cuanto a su percepción del dominio de herramientas multimedia en el cuestionario inicial y el final.

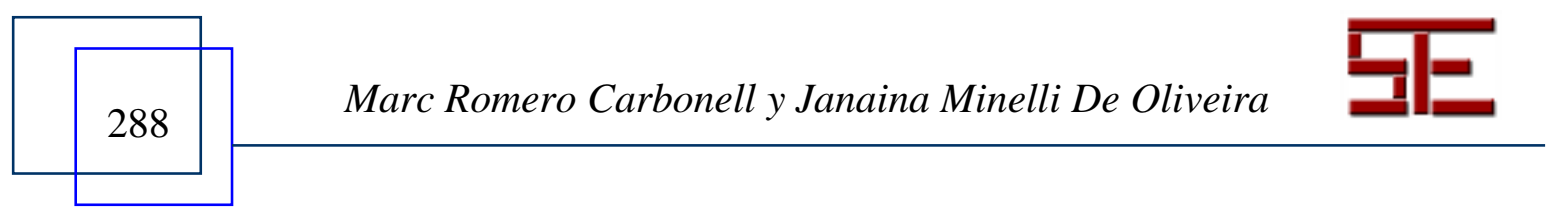




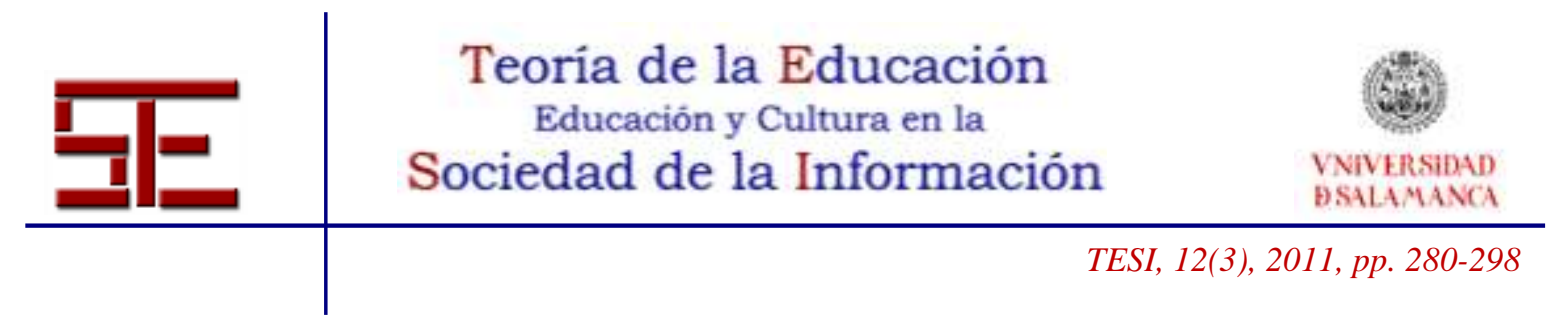

\begin{tabular}{|l|l|l|} 
& $\mathrm{F}(1,53)$ & $\mathrm{p}$ \\
\hline Edición de imágenes & 1,819 & $\mathrm{p}>.05$ \\
Edición de audio & 1,766 & $\mathrm{p}>.05$ \\
Edición de vídeo & 4,861 & $\mathrm{p}<.04$
\end{tabular}

Tabla 2: Resultados del análisis MANOVA entre los ítems referentes a la percepción del dominio las herramientas de edición multimedia y la edad de los sujetos. Cuestionario inicial.

Tal y como se muestra en la tabla 2, no existe una relación significativa entre la edad y la percepción de los alumnos sobre el dominio de las herramientas de edición multimedia. La única excepción es su percepción sobre el dominio de las herramientas de vídeo; aun así, este hecho no se ve ratificado por las medias y la moda ya que se sitúan cerca de 1 en los dos grupos (un 44\% de los alumnos nacidos después de 1982 puntúan su dominio con un 1; un 34, $61 \%$ en el caso de los alumnos mayores), por lo que la relación entre dichas variables se muestra a un nivel bajo.

\begin{tabular}{|l|l|l|} 
& $\mathrm{F}(1,53)$ & $\mathrm{p}$ \\
\hline Edición de imágenes & 0,297 & $\mathrm{p}>.05$ \\
Edición de audio & 1,323 & $\mathrm{p}>.05$ \\
Edición de vídeo & 7,593 & $\mathrm{p}<.03$
\end{tabular}

Tabla 3: Resultados del análisis MANOVA entre los ítems referentes a la percepción del dominio las herramientas de edición multimedia y la edad de los sujetos. Cuestionario final.

En el caso del cuestionario final, la situación se mantiene (Tabla 3), aunque la significación parece aumentar en el caso de la edición de vídeo, de hecho, las modas indican que, en este caso, la edición de vídeo ha tenido un mayor impacto en el caso de los alumnos más jóvenes (un 30\% de los alumnos valoran dicho dominio con un 4 , mientras que, en el caso de los alumnos mayores, este ítem es valorado con un 1 por el $42,9 \%$ de los alumnos). En el caso de las otras aplicaciones, las apreciaciones del análisis MANOVA se cumplen, dado que la moda les sitúa en una puntuación de 4 en edición de imagen en los dos casos y en una puntuación de 3 en el caso de la edición de audio. Por lo tanto, las puntuaciones aumentan respecto al cuestionario inicial (en el que

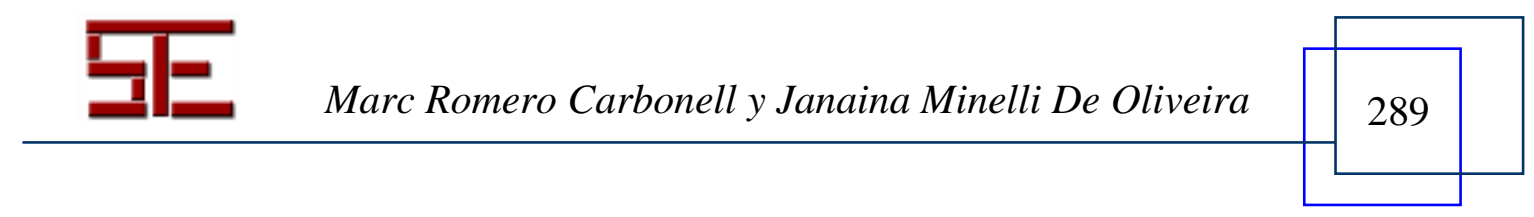




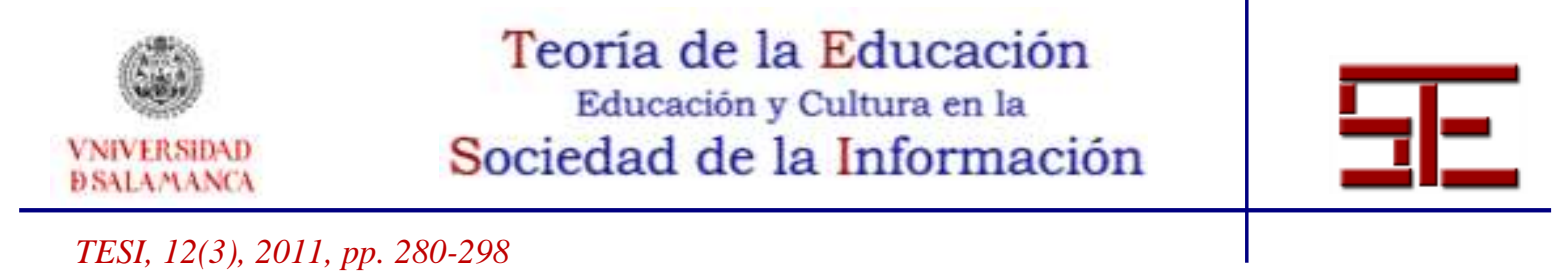

las modas se situaban en 1 en todos los casos); aunque es necesario señalar que durante la asignatura no se dieron orientaciones específicas para el uso de dichas herramientas.

\section{5.- Percepción del dominio de herramientas de comunicación}

\begin{tabular}{|l|l|l|} 
& $\mathrm{F}(1,53)$ & $\mathrm{p}$ \\
\hline Correo electrónico & 0,015 & $\mathrm{p}>.05$ \\
Foros de debate & 1,383 & $\mathrm{p}>.05$ \\
Listas de distribución & 0,001 & $\mathrm{p}>.05$ \\
Programas de chat y videoconferencia & 11,877 & $\mathrm{p}<.01$
\end{tabular}

Tabla 4: Resultados del análisis MANOVA entre los ítems referentes a la percepción del dominio de las herramientas de comunicación y la edad de los sujetos. Cuestionario Inicial.

\begin{tabular}{|l|l|l|} 
& $\mathrm{F}(1,53)$ & $\mathrm{p}$ \\
\hline Correo electrónico & 0,047 & $\mathrm{p}>.05$ \\
Foros de debate & 0,067 & $\mathrm{p}>.05$ \\
Listas de distribución & 0,471 & $\mathrm{p}>.05$ \\
Programas de chat y videoconferencia & 3,087 & $\mathrm{p}>.05$
\end{tabular}

Tabla 5: Resultados del análisis MANOVA entre los ítems referentes a la percepción del dominio de las herramientas de comunicación y la edad de los sujetos. Cuestionario final.

Tal y como se muestra en la Tabla 4, únicamente se muestra algún grado de significación en la relación con la edad de los alumnos en su valoración del dominio de los programas de chat y videoconferencia. Tal y como ya indica el valor de MANOVA, podemos observar un importante grado de significación respecto al resto de ítem. Este hecho queda confirmado por las frecuencias, dado que un $40 \%$ de los alumnos nacidos a partir de 1982 puntuaron dicho ítem con un 6 y un 33\% de los alumnos nacidos antes lo hicieron con un 2.

En cuanto al cuestionario final (Tabla 5), se puede observar un cambio en la valoración del dominio de programas de chat con o sin videoconferencia, hecho que queda ratificado con las modas: un 55,9\% de los estudiantes más jóvenes puntúan su dominio con un 5, mientras que, en el caso de los alumnos mayores, un 30\% lo hace con un 5, por lo que, aunque no exista una significación estadística de la relación de dicho ítem con la edad, las modas indican que los estudiantes más jóvenes siguen sintiéndose más

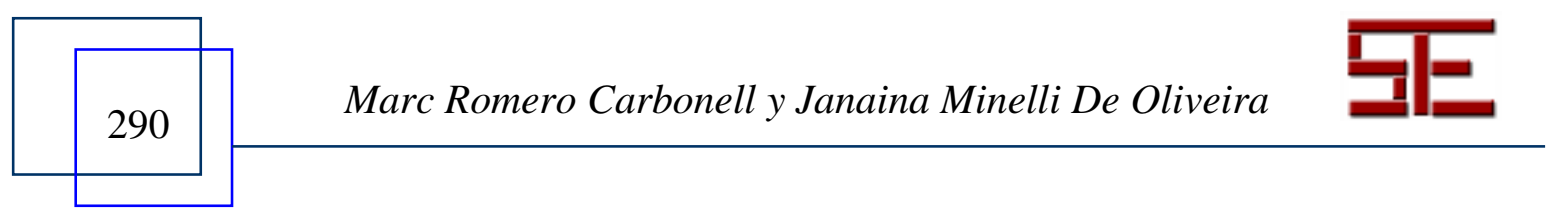




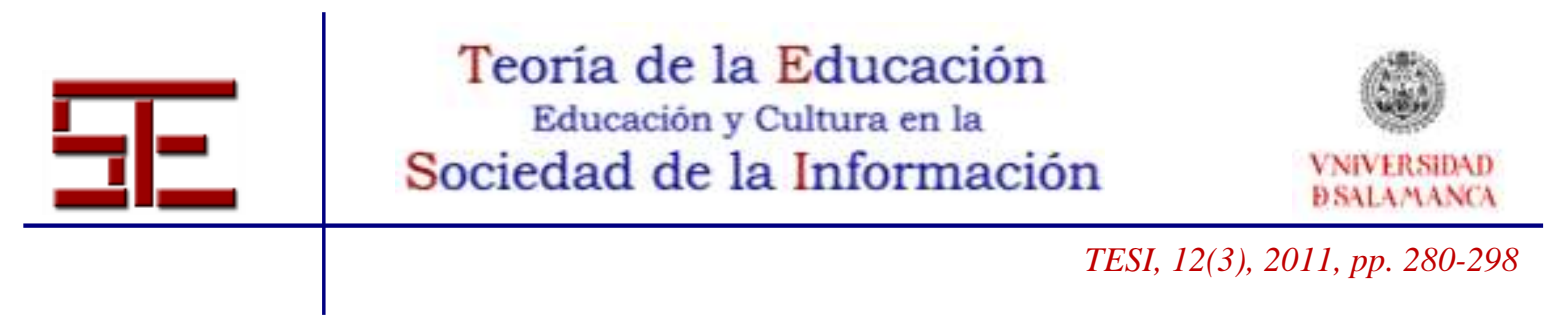

cómodos con su uso. El cambio en la significación estadística de la relación con la edad de este último ítem no puede atribuirse a las actividades llevadas a cabo en nuestra asignatura, dado que no se les pidió explícitamente que usaran este tipo de aplicaciones.

3.6.- Percepción del dominio de herramientas web 2.0

En cuanto al uso y dominio de herramientas web 2.0, el análisis MANOVA muestra los siguientes resultados:

\begin{tabular}{|l|l|l|} 
& $\mathrm{F}(1,53)$ & $\mathrm{p}$ \\
\hline Dominio Blogs & 1,511 & $\mathrm{p}>.05$ \\
$\begin{array}{l}\text { Dominio Wikis } \\
\text { Dominio Redes Sociales (Facebook, }\end{array}$ & 1,389 & $\mathrm{p}>.05$ \\
$\begin{array}{l}\text { Myspace, Ning...) } \\
\text { Dominio Marcadores Sociales (Delicious) y } \\
\text { sindicadores de contenidos (RSS) }\end{array}$ & 2,288 & $\mathrm{p}>.05$ \\
$\begin{array}{l}\text { Dominio Herramientas de trabajo en grupo } \\
\text { (GoogleDocs, Synergia, ...) }\end{array}$ & 3,885 & $\mathrm{p}>.05$
\end{tabular}

Tabla 6: Resultados del análisis MANOVA entre los ítems referentes a la percepción del dominio de las herramientas Web 2.0 y la edad de los sujetos. Cuestionario inicial.

\begin{tabular}{|c|c|c|}
\hline & $\mathrm{F}(1,53)$ & $\mathrm{p}$ \\
\hline Dominio Blogs & ,094 & $\mathrm{p}>.05$ \\
\hline Dominio Wikis & 0,365 & $\mathrm{p}>.05$ \\
\hline Dominio Redes & 1700 & \\
\hline Myspace, Ning...) & 1,904 & $\mathrm{p}<.01$ \\
\hline $\begin{array}{l}\text { Dominio Marcadores sociales (del.icio.us) i } \\
\text { sindicadores de contenidos (RSS) }\end{array}$ & 1,983 & $\mathrm{p}>.05$ \\
\hline $\begin{array}{l}\text { Dominio Trabajo en grupo (Google Docs, } \\
\text { Synergia...) }\end{array}$ & 0,631 & $\mathrm{p}>.05$ \\
\hline
\end{tabular}

Tabla 7: Resultados del análisis MANOVA entre los ítems referentes a la percepción del dominio de las herramientas Web 2.0 y la edad de los sujetos. Cuestionario final.

Tal y como se muestra en la Tabla 6, no existen una relación estadísticamente significativa entre la percepción del dominio de las herramientas web 2.0 y la edad de los alumnos en el caso del cuestionario inicial. Este hecho varía en el caso del

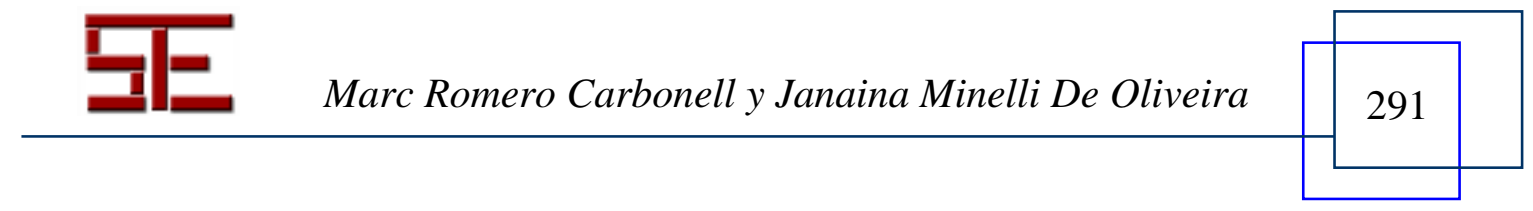




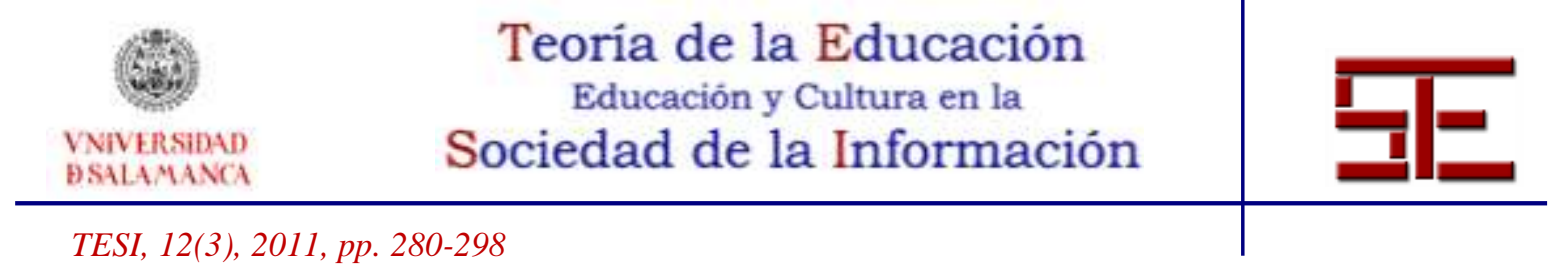

cuestionario final, dado que podemos observar un importante aumento en la relación estadística entre la edad y la percepción del dominio de las redes sociales. Las modas corroboran este hecho, dado que un $44 \%$ de los estudiantes más jóvenes puntúan su dominio con un 5 y un $28,6 \%$ de los estudiantes mayores lo hacen con un 1 . Por lo tanto, al finalizar el curso, los alumnos más jóvenes se sienten más cómodos con el uso de las redes sociales

\section{4.- DISCUSIÓN}

Los datos analizados en este artículo apuntan a que no existe una relación estadística significativa entre la edad de los sujetos de estudio y su percepción acerca del dominio de las TIC. En la línea de Brown y Czerniewicz (2009), los estudiantes más jóvenes no mostraron, en líneas generales, las características que la literatura atribuye a la Generación Net, de hecho, algunos de los alumnos mayores mostraron una mejor percepción en el dominio de las TIC.

Aun así, se ha podido comprobar una preferencia de las nuevas generaciones por los lenguajes audiovisuales o aquellos soportes web en los que el vídeo adquiere una importancia significativa, dado que los datos indican un mayor impacto de dicho medio en los alumnos más jóvenes independientemente de las actividades llevadas a cabo en la asignatura. Teniendo en cuenta que las diferencias entre los dos grupos no son demasiado significativas, no podemos asegurar que los alumnos más jóvenes sean más hábiles en el uso de herramientas multimedia, pero puede deducirse que se sienten más motivados por este tipo de recursos. Lo mismo sucede en el caso del uso de la videoconferencia al inicio de la asignatura, aunque al finalizar el curso, dicha diferencia desaparece. Esta tendencia de los estudiantes más jóvenes a mostrarse más cómodos con los medios audiovisuales en el inicio de la actividad formativa puede ir vinculada a la exposición de los estudiantes más jóvenes a lenguajes audiovisuales en la red. No obstante, en la línea de Bennet, Maton y Kervin (2008), Schulmeister (2009) y Hargittai (2010), no podemos afirmar que se realice una transferencia de dicha motivación al ámbito académico.

Los datos de nuestro estudio coinciden, en la mayoría de aspectos, con los resultados del estudio de Kennedy y otros (2008), al afirmar que no existen diferencias significativas en el uso de las tecnologías web 2.0 entre los dos grupos, sobre todo en el caso del uso de wikis y blogs, aunque los datos indican que la relación entre dichos ítems y la edad de los sujetos es todavía menos significativa en el cuestionario final, aspecto que puede verse explicado por las actividades llevadas a cabo en la asignatura,

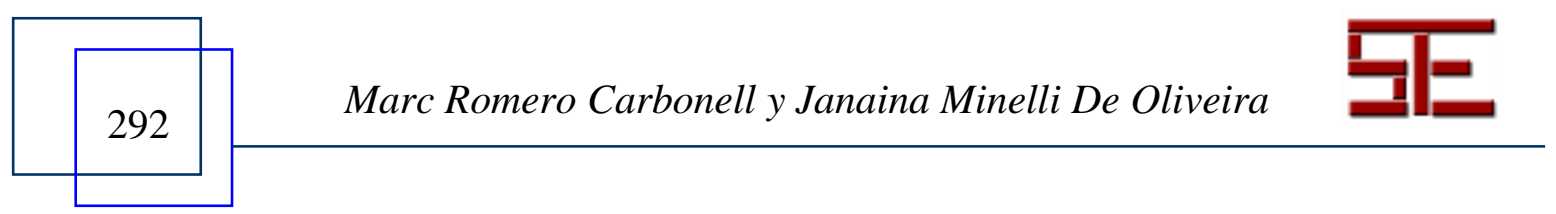




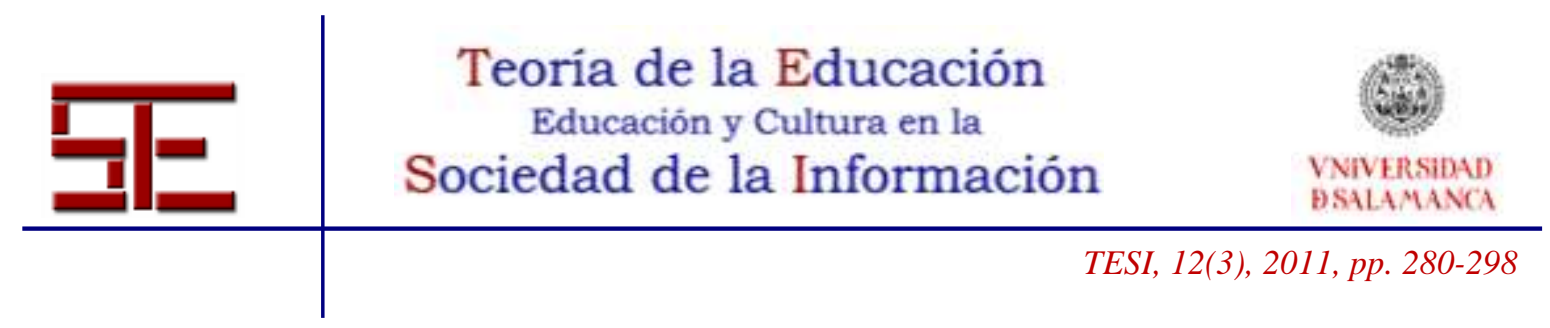

dado que los alumnos utilizaron como referencia el blog de la asignatura ${ }^{1} \mathrm{y}$ diseñaron blogs educativos en las actividades prácticas de la asignatura y utilizaron wikis como material de consulta.

La única excepción se encuentra en el uso de las redes sociales, ítem en el que se observa un importante aumento de la significación estadística de su relación con la edad de los sujetos al finalizar el curso. Dicho cambio puede explicarse a partir de las actividades de la asignatura que, aunque no se trabajaron las redes sociales en las clases prácticas, se dieron a conocer en las sesiones teóricas de curso, teniendo un mayor impacto en los alumnos más jóvenes. Por lo tanto, los sujetos de nuestro estudio coinciden con autores como Tapscott (2009) y Oblinger (2005) en el hecho de mostrar un interés por estar conectados, sintiéndose, en el caso de los alumnos más jóvenes, más cómodos con el hecho de compartir información personal a través de la red.

Aunque se puede observar cierto impacto de la asignatura en la percepción de los alumnos acerca del uso de algunas de las herramientas web 2.0, no sucede lo mismo con su percepción general sobre el dominio de las TIC, ya que no se observan diferencias significativas entre los dos grupos, a excepción de la edición de vídeo y los programas de chat y videoconferencia, pero sin relación directa a las actividades llevadas a cabo en la asignatura.

Nuestro estudio no ha determinado, en líneas generales, evidencias significativas que apoyen las afirmaciones llevadas a cabo por autores como Tapscott (2009) y Oblinger (2005) planteando patrones de aprendizaje radicalmente distintos y nuevas formas de capacitación digital de las nuevas generaciones. En la línea de Romero \& otros (2010), los datos muestran que, en la mayoría de casos, los alumnos mayores suelen sentirse tan cómodos con el uso de las TIC (a excepción de algunas herramientas señaladas) como los alumnos más jóvenes. Por lo tanto, en la línea de Gutiérrez, Palacios y Torrego (2010) al referirse a los estudiantes universitarios españoles, aunque somos conscientes de que los alumnos más jóvenes han entrado en contacto con la tecnología durante más tiempo, el cambio radical al que hace referencia la literatura sobre el tema no se puede aplicar a los estudiantes de la muestra de estudio.

${ }^{1}$ http://tintafrescavlog.blogspot.com/

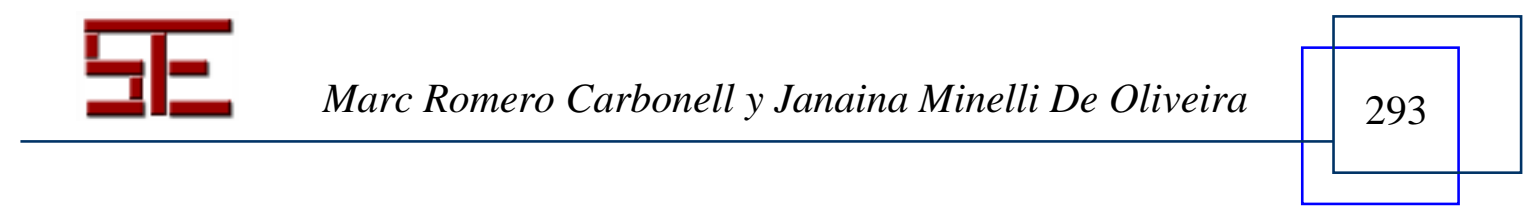




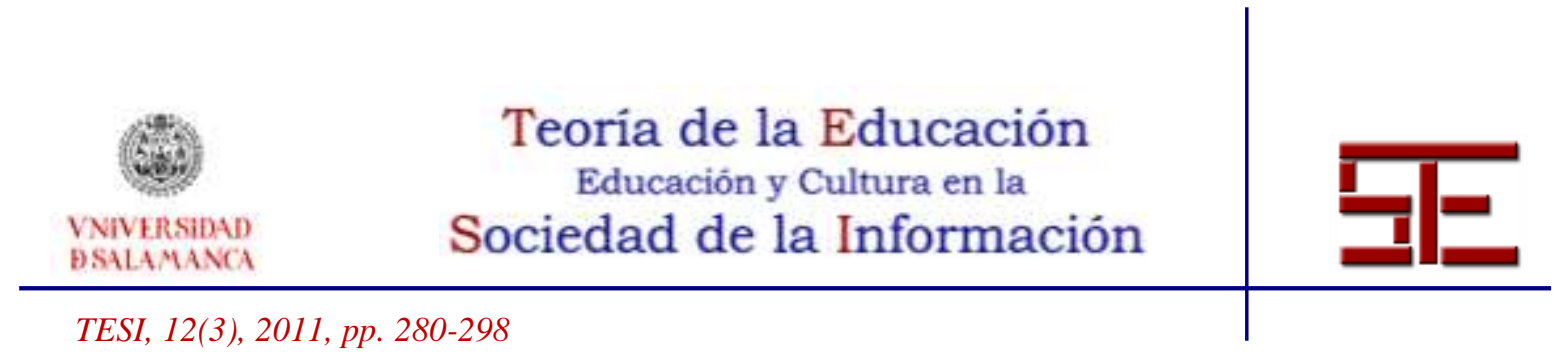

\section{5.- CONCLUSIONES}

Aunque no hemos podido demostrar un mayor dominio de los alumnos más jóvenes de los lenguajes audiovisuales, parece innegable que se sienten más motivados por éstos. Este hecho pone en evidencia la necesidad de la reorientación del foco del sistema educativo contemporáneo para motivar a las nuevas generaciones. La educación formal en distintos niveles, pero más claramente en la secundaria y superior, sigue basándose ampliamente en el lenguaje verbal y en el modo escrito. Los estudiantes, sin embargo, como los datos del presente estudio indican, se sienten enormemente atraídos por las potencialidades comunicativas de los medios audiovisuales, principalmente como herramientas de expresión activa, tal como lo permite la web 2.0, y no por el consumo pasivo fomentado por los medios de comunicación. Si bien es cierto que muchos docentes utilizan actualmente las TIC como componente pedagógico, no es menos cierto que la gran mayoría aún se basa fundamentalmente en los exámenes tradicionales en el momento de evaluar a sus estudiantes. Utilizar las potencialidades creativas de las herramientas audiovisuales que tanto motivan las nuevas generaciones a la creación de mensajes en su campo de aprendizaje es sin duda un reto que el presente estudio demuestra necesario afrontar.

Este estudio implica una reflexión acerca de las implicaciones pedagógicas de la percepción del dominio de las TIC de nuestros estudiantes, de tal forma que, en la línea de Prendes, Castañeda y Gutiérrez (2010), hace necesario reforzar dicha percepción para asegurar un mejor uso de éstas en actividades académicas.

Teniendo en cuenta las características del estudio presentado en este artículo, es necesario señalar sus limitaciones: en primer lugar, pese a que la significación estadística es suficiente para generalizar los resultados a los estudiantes del último curso de magisterio del año analizado, no permite caracterizar el resto de cursos. En segundo lugar, esta investigación se basa en una perspectiva cuantitativa. El análisis cualitativo de los datos permitiría profundizar en aspectos no abordados. Como futuras líneas de investigación, éstas podrían ir encaminadas al análisis cualitativo en pequeña escala de los resultados de aprendizaje de los estudiantes, lo que permitiría contrastar la percepción de su dominio de las TIC con sus blogs y demás recursos creados durante la asignatura.

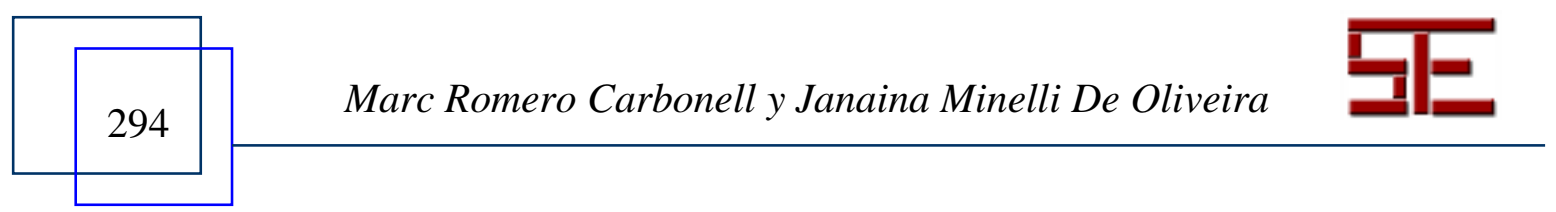




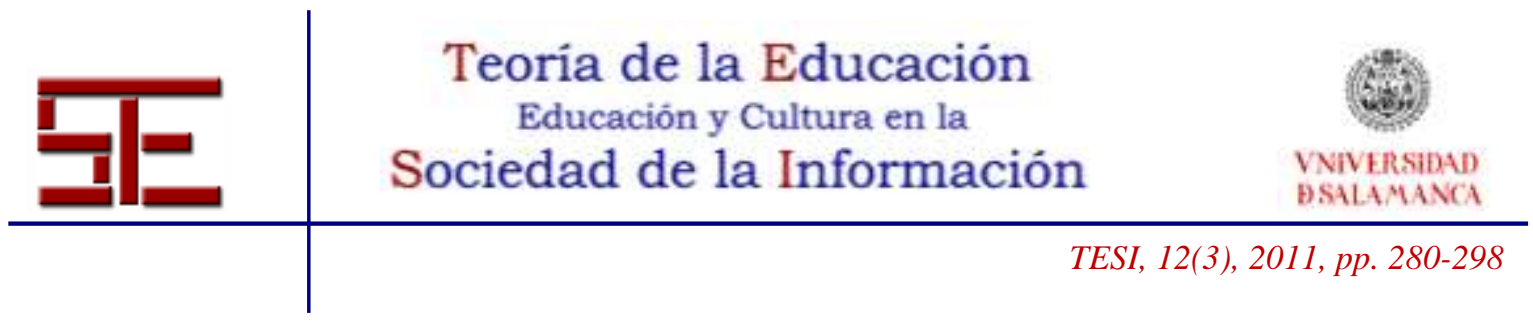

\section{BIBLIOGRAFÍA}

Arnal, J., Del Rincón, D. \& Latorre, A. (1992). Investigación educativa. Fundamentos y metodología. Barcelona: Labor.

Bennett, S., Maton, K. \& Kervin, L. (2008). The 'digital natives' debate: A critical review of the evidence. British Journal of Educational Technology, 39 (5), 775-786.

Berson, I. R. (2003, julio). Making the connection between brain processing and cyberawareness: A Developmental reality. Ponencia presentada en el Simposio Netsafe II, Society, Safety and the Internet Symposium. Auckland, Nueva Zelanda. Extraído el 8 febrero, 2011

de http://www.netsafe.org.nz/Doc_Library/netsafepapers_ileneberson_cyberawareness.pdf

Brown, C. \& Czerniewicz, L. (2009, mayo). The mobile Net Generation: beyond digital apartheid. Ponencia presentada en el Simposio Net Generation, Milton Keynes, Reino Unido. Extraído el 19 abril, 2011 de http://www.idrc.ca/uploads/userS/12759385731Mobile_Net_Generation.pdf.

Bullen, M., Morgan, T. \& Qayyum, A. (2011). Digital learners in higher education: Generation is not the issue. Canadian Journal of Learning Technology, 37(1). Extraído el 1 mayo, 2011 de http://www.cjlt.ca/index.php/cjlt/article/viewFile/550/298.

Cabero, J. (1998). Los Usos de los Medios Audiovisuales, Informáticos y las Nuevas Tecnologías en los Centros Andaluces. Sevilla: Universidad de Sevilla.

Cohen, L. \& Manion, L. (1990). Métodos de investigación educativa. Madrid: La Muralla.

Conole, G. \& otros (2006). LXP: Student experience of technologies. Final report. (Proyecto Learner Experiences of e-Learning). Oxford: Oxford Brookes University. $\begin{array}{lllll}\text { Extraído el } & 10 & \text { octubre, } & 2010 & \text { de }\end{array}$ http://www.jisc.ac.uk/whatwedo/programmes/elearningpedagogy/learneroutcomes.

Cronbach, L. J. (1951). Coefficient alpha and the internal structure of the test. Psychometrika, 16, 297-334.

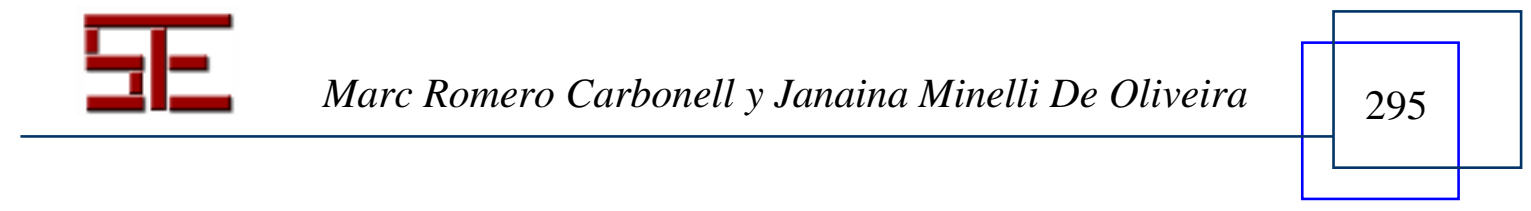




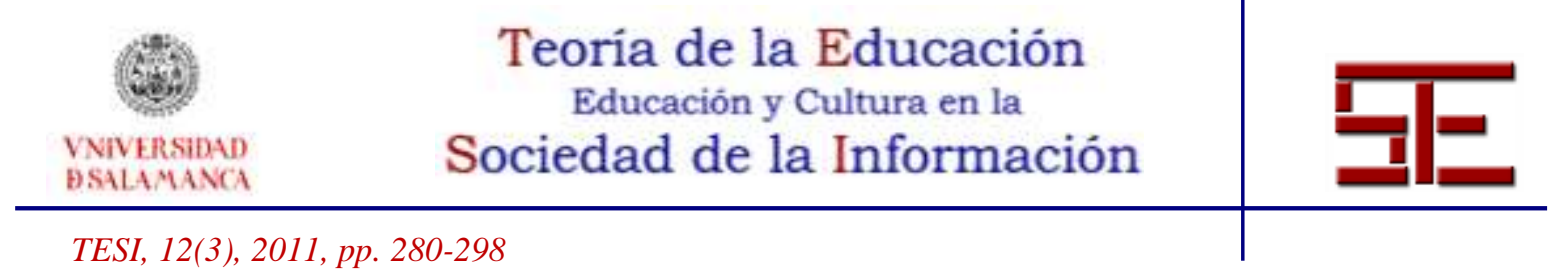

Dede, C. (2005). Planning for neomillenial learning styles [Versión electrónica]. EDUCAUSE Quarterly, 28 (1), 7-12.

Deobold, B. \& otros (1981). Manual de técnica de la investigación educacional. Barcelona: Paidós.

Gall, M. \& otros (1996). Educational research. An introduction. New York: Longman.

Gisbert, M., Lutfi, T. \& Marqués, L. (2004). CREDEFIS: Centro de Recursos Virtual para la Docencia de calidad en Educación Física. Quaderns Digitals, 34. Extraído el 19 diciembre, 2010

de http://www.quadernsdigitals.net/index.php?accionMenu=hemeroteca.VisualizaArticuloI U.visualiza\&articulo_id=7742.

Gutiérrez, A., Palacios, A. \& Torrego, L. (2010). Tribus digitales en las aulas universitarias [Versión electrónica]. Comunicar, 34, 173-181.

Hargittai, E. (2010). Digital Na(t)ives? Variation in Internet Skills and Uses among Members of the "Net Generation" [Versión electrónica]. Sociological Inquiry, 80 (1), 92-113.

Howe, N. \& Strauss, W. (2000). Millennials rising: The next great generation. New York: Vintage.

Karsten, M. C. \& Roth, R. M. (1998). The relationship of computer experience and computer self-efficacy to performance in introductory computer literacy courses [Versión electrónica]. Journal of Research on Computing in Education, 31 (1), 14-24.

Kennedy, G. \& otros. (2008). First year Students' experiences with technology: Are they really digital natives [Versión electrónica]. Australian Journal of Educational Technology, 24(1), 108-122.

Lee, L. (2005). Young people and the Internet from theory to practice. Nordic Journal of Youth Research, 13 (4), 315-326.

Lodico, G. M., \& otros (2006). Methods in Educational research. From Theory to practice. San Francisco: Jossey-Bass.

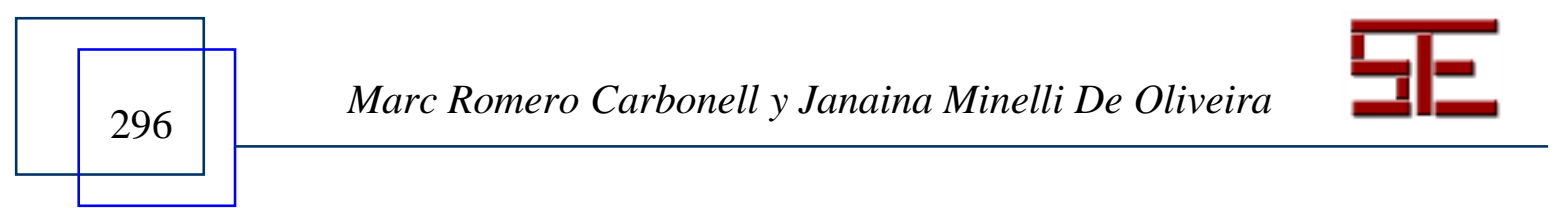




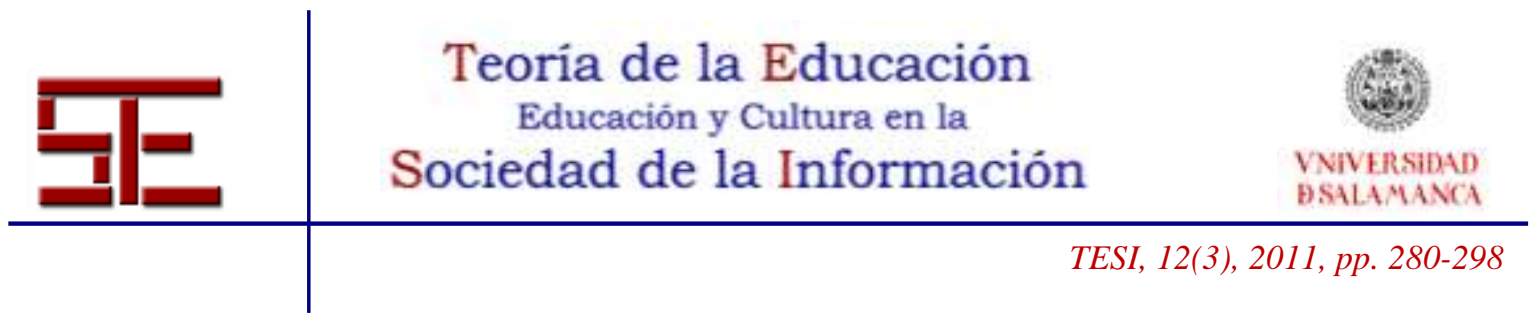

Marcelo, C. (1996). Innovación educativa, asesoramiento y desarrollo profesional. Madrid: Ministerio de Educación y Ciencia.

Margaryan, A. \& Littlejohn, A. (2011). Are digital natives a myth or reality?: Students' use of technologies for learning [Versión electrónica]. Computers and Education, 56 (2) 429-440.

McMillan, J. H. (2004). Educational Research. Fundamentals for the consumer. Boston: Pearson.

- (2006). Understanding an evaluating educational research. Boston: Pearson.

Palfrey, J. \& Gasser, U. (2008). Born digital: understanding the first generation of digital natives. Philadelphia, Pa: Basic Books.

Prendes, P., Castañeda, L. \& Gutiérrez, I. (2010). Competencias para el uso de TIC de los futuros maestros [Versión electrónica]. Comunicar, 35, 175-182.

Prensky, M. (2001). Digital natives, digital immigrants [Versión electrónica]. On the Horizon, 9 (5), 1-6.

Romero, M. (2008). Disseny i avaluació d'un centre virtual de recursos de tecnologia educativa com una eina de formació dels mestres en l'ús de les TIC. Memoria para optar al título de Doctor. Tecnologia Educativa, Universitat Rovira i Virgili, Tarragona, España.

Romero, M. \& otros (2010, junio). Learning in Digital: An Approach to Digital Learners in the UOC Scenario. Comunicación presentada en la Conferencia EDEN, Valencia, España.

Schulmeister, R. (2009). Is There a Net Generation in the House? Dispelling a Mystification. E-learning and education (Eleed), 5. Extraído el 6 febrero, 2011 de http://eleed.campussource.de/archive/5/1587/

Selltiz, C. (1980). Métodos de investigación. Relaciones sociales. Madrid: Rialp.

Selwyn, N. (2009). The digital native: myth and reality [Versión electrónica]. ASLIB proceedings: new information perspectives, 61, (4), 364-379.

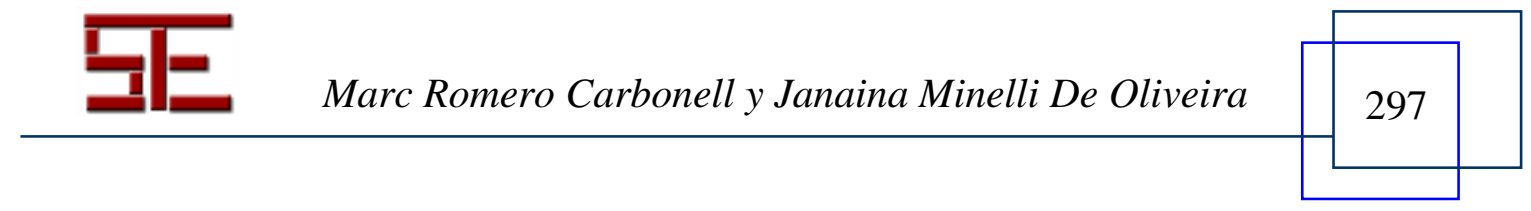




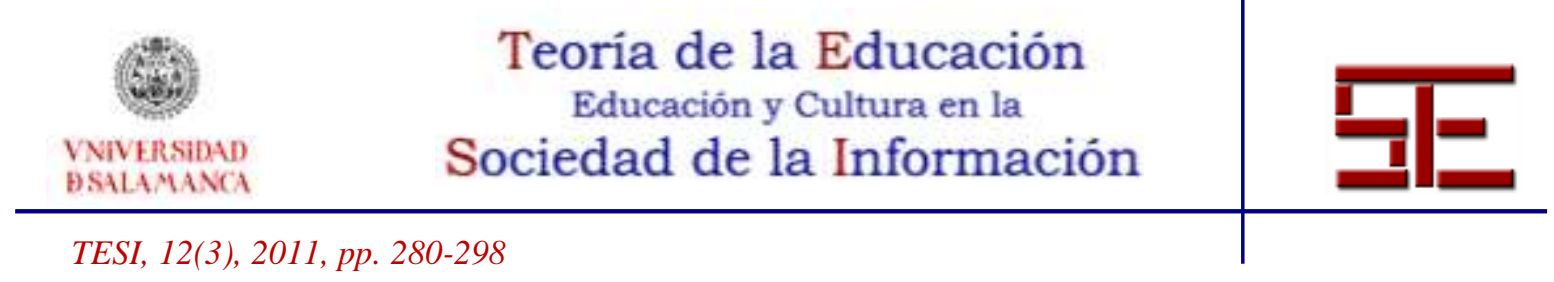

Tapscott, D. (1998). Growing up digital: The rise of the Net generation. New York: McGraw-Hill.

- (2009). Grown up digital: how the Net generation is changing your world. Toronto: Mc Graw-Hill.

Xiaoqing, R. \& otros (2008). Digital natives, digital immigrants: An analysis of age and ICT competency in teacher education [Versión electrónica]. Journal of Educational Computing Research, 38 (3), 235-254.

Para citar el presente artículo puede utilizar la siguiente referencia:

Romero Carbonell, M. y De Oliveira, J. M. (2011). La generación net se tambalea: percepción del dominio de las TIC de estudiantes de magisterio. Revista Teoría de la Educación: Educación y Cultura en la Sociedad de la Información. 12(3), 280-298 [Fecha de consulta: dd/mm/aaaa].

http://campus.usal.es/ revistas_trabajo/index.php/revistatesi/article/view/8492/8585 